\title{
Risk Aversion and Incentive Compatibility with Ex Post Information Asymmetry
}

\author{
Martin F. Hellwig \\ University of Mannheim
}

December 15, 1998

\begin{abstract}
The paper extends Diamond's (1984) analysis of financial contracting with information asymmetry ex post and endogenous "bankruptcy penalties" to allow for risk aversion of the borrower. The optimality of debt contracts, which Diamond obtained for the case of risk neutrality, is shown to be nonrobust to the introduction of risk aversion. This contrasts with the costly state verification literature, in which debt contracts are optimal for risk averse as well as risk neutral borrowers.

Key Words: Debt Contracts, Risk Sharing under Asymmetric Information

Journal of Economic Literature Classification Numbers: D82, G32
\end{abstract}

\section{Introduction}

Under a standard debt contract, a borrower's obligation to his financiers is independent of his actual returns or his ability to pay. If he cannot fulfil the obligation, he goes bankrupt, and the financiers may confiscate his remaining assets. The use of such contracts is commonly explained by differences in information of the borrower and his financiers about outcomes. If the borrower's obligation is independent of his own returns, it is easy for financiers to determine whether the obligation is being fulfilled or not. If the borrower's obligation depends on his returns, financiers have to ascertain what these returns actually are. This may be difficult or costly; the use of debt avoids this difficulty.

There are two distinct formalizations of this argument. The costly state verification approach of Townsend (1979) or Gale and Hellwig (1985) assumes that the information asymmetry can be lifted if resources are spent to provide the financier with information about the borrower's true ability to pay. If all participants are risk neutral, an optimal contract provides for such costly state verification if and only if the borrower cannot pay the prescribed amount; the borrower's remaining assets are then confiscated. This is interpreted as "bankruptcy". 
In contrast, Diamond (1984) assumes that the information asymmetry cannot be lifted at all. It is however possible to use nonpecuniary "bankruptcy penalties" to discourage the borrower from claiming that he cannot repay the financiers. These penalties are chosen endogenously. Their magnitude is made to depend on the amount by which the borrower's payment falls short of his debt service obligation. If all participants are risk neutral, an optimal contract in this setting also takes the form of a standard debt contract.

The apparent similarity of the two approaches disappears if the assumption of risk neutrality is dropped. If the borrower is risk averse and only financiers risk neutral, the costly state verification approach still yields a modified version of a debt contract. In this modified version, the debtor has a state-independent debt service obligation, and state verification occurs if and only if he fails to fulfil this obligation. In the event of "bankruptcy", i.e., when state verification occurs, the borrower's assets are not entirely confiscated: He is left with a positive living allowance. This living allowance is the same in all "bankruptcy" states, providing an element of insurance against the borrower's return risk across bankruptcy states (Townsend (1979), Gale and Hellwig (1985)). Under the condition of Innes (1990), that there must be no incentivefor the borrower to destroy returns before the financiers get to verify anything, the optimal level of this living allowance in the event of bankruptcy is actually equal to the lowest nonbankruptcy consumption of the borrower, i.e., the consumption he has if he can barely fulfil his debt service obligation. ${ }^{1}$

In contrast, the present paper shows that in Diamond's (1984) approach the optimality of debt contracts is not generally robust to the introduction of risk aversion. The underlying incentive considerations are significantly more complex, and an optimal incentive compatible contract should not be expected to have a simple mathematical form. The nonlinearity of the borrower's utility function implies that the nonpecuniary "bankruptcy penalty" that is required to discourage the borrower from underreporting his ability to pay will itself be given by a nonlinear function of the amount of underreporting. Moreover, an optimal contract will involve an element of risk sharing as well as finance. These two considerations interact in such a way that an optimal incentive-compatible contract will typically not take the form of a debt contract, even a debt contract with a positive living allowance.

The difference is illustrated in Figures 1 and 2. The heavy line in Figure 1 exhibits the relation between the return realization $y$ of the borrower and his consumption $c(y)$ under a standard debt contract, with $c(y)=0$ for $y$ below the repayment obligation $\hat{y}$ and $c(y)=y-\hat{y}$ when $y$ exceeds the repayment obligation. The dashed line in Figure 1 exhibits the same relation under a debt

\footnotetext{
${ }^{1}$ As pointed out by Garino and Simmons (1998), optimal contracting in the simple Townsend-Gale-Hellwig model with risk aversion requires that the bankruptcy living allowance have a marginal utility equal to the expected marginal utility of the borrower's consumption in nonbankruptcy states. This implies that the bankruptcy living allowance exceeds the lowest nonbankruptcy consumption level of the borrower. The Innes incentive condition eliminates this possibility, because in "bad nonbankruptcy states" the borrower must not want to destroy output in order to get into bankruptcy and avail himself of the bankruptcy living allowance.
} 
contract with a living allowance $\varepsilon>0$ in the event of "bankruptcy". In contrast, Figure 2 exhibits the relation between $y$ and $c(y)$ under an optimal contract à la Diamond (1984) when (i) financiers are risk neutral, (ii) the borrower exhibits constant relative risk aversion, and (iii) the ex ante distribution of returns is uniform over some interval $[0, Y]$. For high values of $y$, the dependence of $c(y)$ on $y$ looks similar in all three cases, but for low values of $y$, contracting à la Diamond (1984) with risk aversion looks quite different from any form of debt. This suggests that Diamond's model of incentive contracting with endogenous "bankruptcy penalties" is rather less closely related to the costly state verification literature than the parallel results on the optimality of standard debt contracts under risk neutrality would seem to indicate.

I came across these findings when I wanted to extend Diamond's (1984) analysis of financial intermediation to allow for risk aversion of the potential financial intermediaries. Diamond (1984) had used his result on the optimality of debt contracts as an ingredient in the analysis of the conditions under which financial intermediation is efficient in the sense that the overall agency costs of intermediated finance are less than the agency costs of direct finance even though intermediation lengthens the chain of transactions. This analysis involves a diversification argument, which makes essential use of the assumption that intermediaries are risk neutral and raises the question of robustness to the introduction of risk aversion. On the way to answering this question, I found that risk aversion complicates not only the diversification argument for financial intermediation, but also the underlying model of incentive contracting. This latter complication is studied here; on the basis of this analysis, the viability of financial intermediation with risk aversion is studied in a companion paper (Hellwig (1998)). That paper shows that the central results of Diamond (1984) on diversification across borrowers as a basis for intermediation are indeed robust to the introduction of risk aversion.

In the following, Section 2 develops the basic model of incentive contracting with ex post information asymmetry and endogenous bankruptcy penalties for a risk averse borrower. Section 3 discusses optimal contracts and explains the economics underlying the contract exhibited in Figure 2. Proofs are presented in the Appendix.

\section{$2 \quad$ Ex Post Information Asymmetry and Incen- tive Contracting with Nonlinear Utility}

Like Diamond (1984), I consider the financing of a venture that requires a fixed investment $I>0$ and bears a random return $\tilde{y}$. The random variable $\tilde{y}$ has a probability distribution $G$ with a density $g$, which is continuous and strictly positive on the interval $[0, Y]$. The expected return of the venture is strictly greater than the cost $I$, i.e.,

$$
\int_{0}^{Y} y d G(y)>I
$$


The owner/manager of the venture, with own funds $w \geq 0$, wants to raise external finance, either because his funds are less than the investment outlay, or because he wants to share the risk of his venture with others.

Outside financiers know the return distribution $G$, but - in contrast to the entrepreneur - they are unable to observe the realizations of the return random variable $\tilde{y}$. The agency problems caused by this information asymmetry can be reduced through the use of nonpecuniary penalties as a device to discourage misreporting of return realizations. These penalties are determined endogenously as part of the finance contract and moreover they can be made to depend on the entrepreneur's report about his return realization and his actual payment to his financiers.

A finance contract is represented by a number $L$ indicating the funds provided by outside financiers and by two functions $r($.$) and p($.$) such that for any$ $z \in[0, Y], r(z)$ is the payment to financiers and $p(z) \geq 0$ is the nonpecuniary penalty the entrepreneur suffers when he reports that his return realization is equal to $z$. With outside funds $L$, his own financial contribution to his project is $E=I-L \leq w$. Any excess of $w$ over $E$ is invested in an alternative asset, which is safe and has a gross rate of return equal to one.

Given a finance contract $(L, r(),. p()$.$) , the entrepreneur's consumption is$ $w+L-I+y-r(z)$ if the true return realization is $y$ and the reported return realization is $z$; the corresponding payoff realization is $u(w+L-I+y-r(z))-$ $p(z)$. A contract $(L, r(),. p()$.$) is said to be feasible if L \geq I-w$ and moreover,

$$
w+L-I+y-r(y) \geq 0
$$

for all $y \in[0, Y]$, so the entrepreneur's consumption is never negative. A contract $(L, r(),. p()$.$) is said to be incentive compatible if it is feasible and moreover$

$$
u(w+L-I+y-r(y))-p(y) \geq u(w+L-I+y-r(z))-p(z)
$$

for all $y \in[0, Y]$ and all $z \in[0, Y]$ such that $w+L-I+y \geq r(z)$, so he has no incentive to misreport his return realization.

The utility function $u($.$) is assumed to be strictly increasing and strictly$ concave as well as twice continuously differentiable on $\Re_{++}$; moreover, $u(0)=$ $\lim _{c \rightarrow 0} u(c)$, with the usual conventions when $\lim _{c \rightarrow 0} u(c)=-\infty$, e.g., when $u()=.\ln ($.$) . Given these assumptions, standard arguments from incentive the-$ ory yield:

Proposition 1 A finance contract $(L, r(),. p()$.$) satisfying (2) for all y \in[0, Y]$ is incentive compatible if and only if:

(i) the function $r($.$) is nondecreasing on [0, Y]$ and

(ii) for all $y \in[0, Y]$,

$$
p(y)=p(Y)+\int_{y}^{Y} u^{\prime}(w+L-I+x-r(x)) d r(x)
$$


Condition (4) shows that for a given loan size $L$ and repayment function $r($.$) ,$ incentive compatibility determines the penalty function $p($.) up to a constant of integration, $p(Y)$. If $r($.$) is differentiable, this condition is actually equivalent$ to the differential equation

$$
\frac{d p}{d y}=-u^{\prime}(w+L-I+y-r(y)) \frac{d r}{d y},
$$

showing that as the return realization $y$ goes down, the penalty $p(y)$ goes up at a rate which depends on the rate $\frac{d r}{d y}$ at which the payment $r(y)$ goes down as $y$ goes down.

As an illustration, consider the class of finance contracts $(L, r(),. p()$.$) such$ that

$$
r(y)=w+L-I+\min (y, \hat{y})-\varepsilon
$$

for some fixed $\varepsilon \geq 0, \hat{y} \in(0, Y)$, and all $y \in[0, Y]$. Such contracts can be interpreted as debt contracts with a minimum living allowance $\varepsilon$. The amount $w+L-I+\hat{y}-\varepsilon$ represents a return-independent debt service obligation. If the entrepreneur can meet this obligation he does so and retains the excess of his actual return $y$ over $\hat{y}$ as well as $\varepsilon$. If he cannot meet the obligation $w+L-I+\hat{y}-\varepsilon$, he defaults and retains just the minimum living allowance $\varepsilon$. If the minimum living allowance is zero, (6) is the repayment function for a standard debt contract as studied by Diamond (1984) or Gale and Hellwig (1985).

By (6), a debt contract has $\frac{d r}{d y}=0$ if $y>\hat{y}$ and the obligation $w+L-I+\hat{y}-\varepsilon$ is met, but $\frac{d r}{d y}=1$ if $y<\hat{y}$ and the entrepreneur defaults on his obligation. Thus condition (5) entails $\frac{d p}{d y}=0$ if $y>\hat{y}$, and $\frac{d p}{d y}=-u^{\prime}(\varepsilon)$ if $y<\hat{y}$; condition (4) reduces to:

$$
p(y)=p(Y)+\max (\hat{y}-y, 0) u^{\prime}(\varepsilon) .
$$

If $\varepsilon=0$ and $u^{\prime}(0)=1,(7)$ is exactly the condition that Diamond (1984) gives for the incentive compatibility of a standard debt contract for the case of risk neutrality ${ }^{2}$, requiring that the difference $p(y)-p(Y)$ be just equal to the amount of money that the entrepreneur saves by paying $r(y)$ rather than $r(Y)$. For $y \in[\hat{y}, Y]$ of course, (6) implies $r(y)=r(Y)$ and hence $p(y)=p(Y)$. If $u^{\prime}(\varepsilon) \neq 1$, the money gain $r(\hat{y})-r(y)=\hat{y}-y$ from reporting $y<\hat{y}$ rather than $\hat{y}$ under the repayment function (6) has to be weighted by $u^{\prime}(\varepsilon)$ so as to as to make the penalty $p(y)$ commensurate with the utility gain from reporting

\footnotetext{
${ }^{2}$ Risk neutrality is not compatible with the assumption that $u($.$) is strictly concave. A$ careful analysis of the proof of Proposition 1 shows that this assumption is used only to establish that incentive compatibility entails weak monotonicity of $r($.$) . The other parts$ of Proposition 1, i.e., the sufficiency of (i) and (ii) and the necessity of (ii) for incentive compatibility, go through even if $u($.$) is merely weakly concave.$
} 
$y$ rather than $\hat{y}$ and paying $r(y)$ rather than $r(\hat{y})$. (If $u^{\prime}(0)$ is very large, this militates against the use of a standard debt contract as opposed to one with a minimum living allowance $\varepsilon>0$.)

Turning to the choice between contracts, I note that the entrepreneur's expected payoff from an incentive-compatible contract $(L, r(),. p()$.$) is equal to:$

$$
\int_{0}^{Y} u(w+L-I+y-r(y)) d G(y)-\int_{0}^{Y} p(y) d G(y)
$$

Upon using (4) to substitute for $p(y)$ and integrating the resulting double integral by parts, one finds that this is equal to

$$
\begin{aligned}
& \int_{0}^{Y} u(w+L-I+y-r(y)) d G(y) \\
& -\int_{0}^{Y} u^{\prime}(w+L-I+y-r(y)) G(y) d r(y)-p(Y)
\end{aligned}
$$

As for the financiers, I assume that there are enough of them dividing the uncertain return $r(\tilde{y})$ among each other so that they assess the contract $(L, r(),. p()$.$) as if they were risk neutral. They are only concerned as to whether$ the expected gross return $\int_{0}^{Y} r(y) d G(y)$ is enough to cover the opportunity cost of their putting up the funds $L$. From their perspective, an incentive-compatible finance contract $(L, r(),. p()$.$) is acceptable, if and only if$

$$
\int_{0}^{Y} r(y) d G(y) \geq L
$$

Condition (1) ensures that the set of acceptable contracts is nonempty. An acceptable incentive-compatible finance contract $(L, r(),. p()$.$) is called optimal$ if it maximizes the entrepreneur's expected payoff (8), respectively (9), over the set of all acceptable incentive-compatible contracts.

In the remainder of the paper, I study the properties of optimal incentivecompatible contracts. I begin with the observation that, as shown in (9), the entrepreneur wants $p(Y)$, the penalty he suffers when he reports the maximum possible return, to be as small as possible. As for the financiers, (10) shows that their payoff is independent of $p(Y)$; moreover (4) shows that incentive compatibility hinges on the difference $p(y)-p(Y)$ rather than the level of $p(Y)$. Trivially then one obtains:

Remark 2 Any optimal incentive-compatible contract satisfies $p(Y)=0$. 


\section{Optimal Incentive-Compatible Contracts}

In view of Proposition 1, the problem of finding an optimal incentive-compatible contract is equivalent to the problem of finding a loan size $L \geq I-w$ and a nondecreasing repayment function $r($.$) so as to maximize (9)$, with $p / Y)=0$, subject to the feasibility constraint (2) and the acceptability condition (10). It is convenient to rewrite this problem in terms of the entrepreneur's consumption pattern $c($.$) , where for any y \in[0, Y]$,

$$
c(y):=w+L-I+y-r(y) .
$$

Since (11) implies $d r(y)=d y-d c(y)$, the objective function (9) with $p(Y)=0$ can be rewritten as

$$
\int_{0}^{Y} u(c(y)) g(y) d y-\int_{0}^{Y} u^{\prime}(c(y)) G(y) d y+\int_{0}^{Y} u^{\prime}(c(y)) G(y) d c(y) .
$$

Upon combining the first and the third term and integrating, one can further rewrite this as

$$
u(c(Y))-\int_{0}^{Y} u^{\prime}(c(y)) G(y) d y
$$

The financiers' participation constraint (10) is similarly rewritten as:

$$
\int_{0}^{Y} c(y) d G(y) \leq w-I+\int_{0}^{Y} y d G(y)
$$

Finally, with (4) subsumed in (9), respectively (13), feasibility and incentive compatibility reduce to the requirements that

$$
c(y) \geq 0
$$

and that $r($.$) be nondecreasing or, equivalently, that$

$$
c(y)-c(z) \leq y-z
$$

for all $y, z \in[0, Y]$ such that $y \geq z$. The optimal-contracting problem has thus been reduced to the problem of choosing a function $c($.$) on [0, Y]$ so as to maximize (13) under the constraints (14)-(16).

Proposition 3 Under the maintained assumptions, in particular (1), an optimal incentive-compatible contract exists.

Proposition 4 If $c($.$) corresponds to an optimal incentive-compatible contract,$ then $c($.$) is continuous on (0, Y]$. Moreover $c(Y)>0$ and $c(y)=c(Y)+y-Y$ for $y$ sufficiently close to $Y$. 
An optimal contract always exhibits the feature of a debt contract whereby for high realizations of the borrower's return a further increase in his return leaves his payment to the financiers unaffected, i.e., all of this increase serves to raise his consumption. This reflects the prominence of $u(c(Y))$ in $(13)$ : For return levels close to $Y$, it is important to have $c(y)$ increase as much as possible with $y$ so as to make $c(Y)$ and hence $u(c(Y))$ large. Accordingly the consumption patterns in Figures 1 and 2 all have a slope $\frac{d c}{d y}$ equal to one when the return level $y$ is close to the upper bound $Y$.

In contrast, for low realizations of the borrower's return, an optimal contract in the presence of risk aversion does not always exhibit the feature of a debt contract that lenders confiscate everything "in the event of bankruptcy". Indeed Propositions 6 - 8 below show that for many specifications of the borrower's utility function the repayment owed to lenders is insensitive to the borrower's return realization when the latter is low as well as when it is high, and the borrower's consumption $c(y)$ is bounded away from zero.

The analysis uses control-theoretic methods. If $c($.$) was known to be abso-$ lutely continuous, the problem of maximizing (13) under the constraints (14) (16) could be formulated as a standard optimum-control problem with control $v(y):=\frac{d c}{d y}(y)$ and $(16)$ equivalent to the requirement that $v(y) \leq 1$ for all $y$. The assumptions here do not actually guarantee this. Even so, the consumption pattern induced by an optimal contract must satisfy a suitable analogue of Pontryagin's conditions. This is the point of:

Proposition 5 Let $c($.$) correspond to an optimal incentive-compatible contract.$ Then there exist a scalar $\mu>0$ and a continuously differentiable real-valued function $\psi($.$) on [0, Y]$ such that for all $y \in[0, Y]$,

$$
\begin{gathered}
\frac{d \psi}{d y} \leq u^{\prime \prime}(c(y)) G(y)+\mu g(y), \text { with equality if } c(y)>0 \\
\psi(y) \geq 0, \text { with equality unless in a neighbourhood of } y \\
c(.) \text { is continuously differentiable with } \frac{d c}{d y}=1 \\
\psi(Y)=u^{\prime}(c(Y)) \\
\psi(0)=0 .
\end{gathered}
$$

If $u^{\prime \prime}($.$) is a strictly increasing function, these conditions are sufficient as well$ as necessary for $c($.$) to maximize (13) under the constraints (14)-(16); in this$ case, the optimal contract is unique in the sense that consumption patterns corresponding to different optimal contracts all coincide on $(0, Y]$. 
It is instructive to consider the case of constant absolute risk aversion. In this case, as in the more general case of nonincreasing absolute risk aversion, $u^{\prime \prime}($. is automatically a strictly increasing function, so the maximand (13) is strictly concave in $c($.$) , and the last part of Proposition 5$ applies, i.e., the consumption pattern corresponding to an optimal contract is completely characterized by the Pontryagin conditions (17)-(20). This yields:

Proposition 6 Assume that $u($.$) exhibits constant absolute risk aversion, i.e.,$ that $u(c) \equiv-e^{-\delta c}$ for some $\delta>0$. Assume further that the distribution $G($. is uniform, i.e., that $g(y) \equiv 1 / Y$, and let $c(., \delta)$ be the consumption pattern corresponding to an optimal incentive-compatible contract.

(a) If $\delta$ is sufficiently close to zero, then $c(., \delta)$ has the form shown in Figure $1, i . . e$.

$$
c(y, \delta)=\max (w-I, 0)+\max (0, y-\hat{y}),
$$

where $\hat{y} \in[0, Y)$ is chosen so that (14) holds with equality.

(b) If $\delta$ is sufficiently large, then $c(., \delta)$ has the form shown in Figure 2, i.e., $c(0, \delta)>0$, and there exist $y_{1}(\delta), y_{2}(\delta) \in(0, Y)$ such that

$$
\frac{d c}{d y}=1 \text { if } y<y_{1}(\delta) \text { or } y>y_{2}(\delta)
$$

and

$$
c(y, \delta)=c\left(y_{1}, \delta\right)+\delta^{-1}\left[\ln y-\ln y_{1}(\delta)\right] \text { if } y_{1}(\delta) \leq y \leq y_{2}(\delta)
$$

Moreover as $\delta$ goes out of bounds, $y_{1}(\delta)$ converges to zero, $y_{2}(\delta)$ converges to $Y$, and $c(., \delta)$ converges to the constant function with value $w-I+\int y d G(y)$, uniformly on $[0, Y]$.

With constant absolute risk aversion, a standard debt contract is optimal if risk aversion is close to zero, but not if risk aversion is large. To understand the economics behind this result, go back to the borrower's objective function as specified in (12) and rewrite this in the form

$$
\int_{0}^{Y} u(c(y)) g(y) d y-\int_{0}^{Y} u^{\prime}(c(y)) G(y)(1-v(y)) d y
$$

where, for any $y, v(y):=\frac{d c}{d y}$. (In the constellation of Proposition 6 this is actually legitimate.) In the case of risk neutrality, with $u(c) \equiv c$ and $u^{\prime}(c) \equiv 1$, (24) simplifies to

$$
\int_{0}^{Y} c(y) g(y) d y-\int_{0}^{Y} G(y)(1-v(y)) d y .
$$


An optimal contract must obviously satisfy (14) with equality. In the case of risk neutrality, this fixes the first term in (25) as $w-I+\int y d G$, regardless of any other aspect of the consumption pattern $c($.$) . The shape of c($.$) is then chosen$ solely with a view to minimizing the expected value $\int_{0}^{Y} G(y)(1-v(y)) d y$ of nonpecuniary penalties. If $w \geq I$, this requires $v(y)=1$ for all $y$; if $w<I$, it requires that $v(y)=1$ if $G(y)$ is large and $v(y)=c(y)=0$ if $G(y)$ is small. This explains Diamond's (1984) result on the optimality of standard debt under risk neutrality. ${ }^{3}$

The appearance of the weights $G(y)$ in the expressions for expected nonpecuniary penalties in (25) reflects the fact that the incentive compatibility condition (4) relates changes in $p($.$) to changes in r($.$) . If \frac{d r}{d y}=1-v(y)$ is positive over some interval $(y-\Delta, y]$, the increase in penalties as one goes from $y$ to $y-\Delta$ affects the level of penalties not just at $y-\Delta$, but at all return levels $y^{\prime}<y$ (to discourage the entrepreneur from misreporting $y^{\prime}$ instead of $y-\Delta$ ). This explains why the "increase" $d r=(1-v(y)) d y$ enters (25) with the weight $G(y)$ of the set of all return levels less than $y$. Given this appearance of the weights $G(y)$ in (25), under risk neutrality it is desirable to concentrate the deviations of $v(y)$ from one at low levels of $y$.

A simple comparison of (24) and (25) shows that this argument for the optimality of debt contracts is heavily dependent on the assumption of risk neutrality. If the von Neumann-Morgenstern utility function $u($.$) is strictly$ concave, two additional considerations must be taken into account: First, when the borrower is risk averse, the first term in (24) depends on the riskiness as well as the mean of the random variable $c(\tilde{y})=w+L-I+\tilde{y}-r(\tilde{y})$. If $u^{\prime}(c)$ is large when $c$ is close to zero, this militates against $c(\tilde{y})$ being zero with positive probability. Secondly, the weight with which "the increase" $d r$ enters the expected value of the nonpecuniary penalties in the last term in (24) depends on $u^{\prime}(c(y))$ as well as $G(y)$, the point being that the nonpecuniary penalties have to compensate for utility gains from false reporting, not just the money gains. If marginal utility is large, a given money gain from false reporting may translate into a large utility gain, requiring a large penalty to keep the borrower honest. Whereas under risk neutrality, expected nonpecuniary penalties are minimized by concentrating the increases of $r($.$) at those return levels where G(y)$ is small, with risk aversion, they are minimized by concentrating them at those return levels where $u^{\prime}(c(y)) G(y)$ is small. This need not be where $y$ is small.

The consumption pattern in Figure 2 reflects these considerations. In Figure 2 , in contrast to Figure 1, the slope $v(y)=\frac{d c}{d y}$ is equal to one for very low as well as very high values of $y$; this reflects the possibility that the weight $u^{\prime}(c(y)) G(y)$ of the term $(1-v(y))$ in (24) may be large if $c(y)$ is small and $u^{\prime}(c(y))$ is large. In an intermediate range in Figure 2, $v(y)=\frac{d c}{d y}$ lies strictly between zero and one, reflecting a tradeoff at the margin between considerations of risk sharing (calling for a low value of $v(y)$ ), the need to repay the financiers (again calling for a low

\footnotetext{
${ }^{3}$ Alternatively, the reader may observe that under risk neutrality, (13) takes the form $c(Y)-\int G(y) d y$, which is maximal if $c(Y)$ is maximal, which in turn is the case when $c($. takes the form (21), see Lemma 9 in the Appendix.
} 
value of $v(y)$ ) and the desire to keep nonpecuniary penalties low (calling for a high value of $v(y))$.

The important point is that in the presence of risk aversion the finance contract provides for risk sharing as well as finance. Even if $w \geq I$, i.e., if the entrepreneur is able to finance his project on his own, he may still want to bring in an external investor as this enables him to maintain his consumption when project returns are low. He has to pay for this insurance in terms of nonpecuniary penalties, but depending on his risk preferences and on the distribution of returns, he may well find this worthwhile. This is, e.g., always the case in the constellation of Proposition 6 when $\delta$ is large; in this case, regardless of the relation of $w$ and $I$, an optimal incentive-compatible contract will provide the entrepreneur with a consumption pattern close to the nonrandom constant $w-I+\int y d G(y)$.

More generally, for the case of constant absolute risk aversion, Proposition 6 shows that risk sharing considerations play no role if risk aversion is low, but entail the nonoptimality of debt contracts if risk aversion is high. For other utility functions, risk sharing considerations always preclude the optimality of debt contracts if $u^{\prime}(c)$ becomes large as $c$ becomes small, e.g., if the von NeumannMorgenstern utility function exhibits constant relative risk aversion. This is shown in:

Proposition 7 Let $c($.$) correspond to an optimal incentive-compatible contract.$ If $\lim _{c \rightarrow 0} u^{\prime}(c)=\infty$, then $c(y)>0$ for all $y \in(0, Y]$. If $\lim _{c \rightarrow \infty} u^{\prime \prime}(c) c=-\infty$, then $c(0)>0$ and $v(y)=1$ for any $y$ that is sufficiently close to 0 .

Proposition 8 Assume that $u($.$) exhibits constant relative risk aversion, i.e.,$ that $u^{\prime}(y) \equiv c^{\delta-1}$ for some $\delta<1$. Assume further that the distribution function $G($.$) is uniform, i.e., that g(y) \equiv 1 / Y$, and let $c($.$) be the consumption pattern$ corresponding to an optimal incentive-compatible contract. If $w<I$, then $c($. has the form shown in Figure 2, i.e., $c(0)>0$, and there exist $y_{1}, y_{2} \in(0, Y)$ such that

$$
\frac{d c}{d y}=1 \text { if } y<y_{1} \text { or } y>y_{2}
$$

and

$$
c(y)=c\left(y_{1}\right)\left(y / y_{1}\right)^{1 /(2-\delta)} \text { if } y_{1} \leq y \leq y_{2} .
$$

For other specifications of utility and distribution functions, yet more complicated finance contracts may be optimal. To see this, note that if $v(y)=\frac{d c}{d y}$ is not equal to one, then, by (18), the costate variable $\psi($.$) must have a local$ minimum at $y$. If in addition $c(y)>0$, then by (17), one must have

$$
u^{\prime \prime}(c(y)) G(y)+\mu g(y)=0 \text {. }
$$


This equation represents the tradeoff at the margin mentioned above that underlies an interior choice of $v(y)=\frac{d c}{d y}$. If $u^{\prime \prime}($.$) is a strictly increasing function,$ (28) can be rewritten as

$$
c(y)=u^{\prime^{-1}}\left(-\mu \frac{g(y)}{G(y)}\right) .
$$

Using (29), one easily verifies that if $u^{\prime \prime}($.$) is strictly increasing and g(.) / G($.$) is$ nondecreasing, then the consumption pattern $c($.$) that corresponds to an opti-$ mal incentive-compatible contract is nondecreasing. Otherwise, e.g., if $u^{\prime \prime}($.$) is$ increasing and the hazard rate function $g(.) / G($.$) is not everywhere nondecreas-$ ing, $c($.$) may be decreasing somewhere. Moreover, depending on the slope of$ the function $u^{\prime \prime^{-1}}(-\mu g(.) / G()$.$) , the number of switches back and forth between$ intervals where $\frac{d c}{d y}$ takes an interior value and intervals where $\frac{d c}{d y}=1$ may be arbitrarily large. Optimal incentive-compatible contracts are thus very sensitive to the specification of the functions $u^{\prime \prime}($.$) and g(.) / G($.$) .$

\section{A Appendix}

Proof of Proposition 1. Suppose first that a finance contract satisfies conditions (i) and (ii) of the proposition. For any $y \in[0, Y]$ and any $z \in[0, Y]$ such that $r(z) \in w+L-I+y$, one then has, from (4)

$$
\begin{gathered}
u(w+L-I+y-r(y))-p(y)-[u(w+L-I+y-r(z))-p(z)] \\
=-\int_{r(z)}^{r(y)} u^{\prime}(w+L-I+y-r) d r+\int_{z}^{y} u^{\prime}(w+L-I+x-r(x)) d r(x)
\end{gathered}
$$

If $z<y$, concavity of $u($.$) implies u^{\prime}(w+L-I+x-r(x)) \geq u^{\prime}(w+L-I+y-r(x))$ for all $x \in[z, y]$. By the monotonicity of $r($.$) , one then has:$

$$
\begin{aligned}
\int_{z}^{y} u^{\prime}(w+L-I+x-r(x)) d r(x) & \geq \int_{z}^{y} u^{\prime}(w+L-I+y-r(x)) d r(x) \\
& =\int_{r(z)}^{r(y)} u^{\prime}(w+L-I+y-r) d r
\end{aligned}
$$

which means that (A.1) implies (3). Alternatively, if $z>y$, concavity of $u($. implies $u^{\prime}(w+L-I+x-r(x)) \leq u^{\prime}(w+L-I+y-r(x))$ for all $x \in[y, z]$. By the monotonicity of $r($.$) , one then has:$ 


$$
\begin{aligned}
\int_{y}^{z} u^{\prime}(w+L-I+x-r(x)) d r(x) & \leq-\int_{y}^{z} u^{\prime}(w+L-I+y-r(x)) d r(x) \\
& =\int_{r(y)}^{r(z)} u^{\prime}(w+L-I+y-r) d r
\end{aligned}
$$

and again (A.1) implies (3). This shows that any finance contract which satisfies assertions (i) and (ii) in the proposition is incentive-compatible.

Conversely, suppose that a contract $(L, r(),. p()$.$) is incentive-compatible.$ Let $0 \leq y_{1}<y_{2} \leq Y$. Apply the incentive compatibility condition (1) once with $y=y_{2}$ and $z=y_{1}$, and, assuming that $r\left(y_{2}\right) \leq w+L-I+y_{1},{ }^{4}$ once with $y=y_{1}$ and $z=y_{2}$, and add the resulting inequalities.

This yields

$$
\begin{aligned}
& u\left(w+L-I+y_{2}-r\left(y_{2}\right)\right)+u\left(w+L-I+y_{1}-r\left(y_{1}\right)\right) \\
& \geq u\left(w+L-I+y_{2}-r\left(y_{1}\right)\right)+u\left(w+L-I+y_{1}-r\left(y_{2}\right)\right)
\end{aligned}
$$

or, after a rearrangement of terms,

$$
\int_{y_{1}}^{y_{2}} u^{\prime}\left(w+L-I+x-r\left(y_{2}\right)\right) d x \geq \int_{y_{1}}^{y_{2}} u^{\prime}\left(w+L-I+x-r\left(y_{1}\right)\right) d x
$$

Given that $u($.$) is strictly concave, this inequality implies r\left(y_{2}\right) \geq r\left(y_{1}\right)$, proving that $r($.$) is nondecreasing on [0, Y]$.

To prove that the contract also satisfies (4), note that for any $y \in[0, Y]$ and $x<y,(3)$ implies

$$
\begin{aligned}
p(y)-p(x) & \leq u(w+L-I+y-r(y))-u(w+L-I+y-r(x)) \\
& =-\int_{r(x)}^{r(y)} u^{\prime}(w+L-I+y-r) d r \\
& \leq-\int_{r(x)}^{r(y)} u^{\prime}(w+L-I+x-r) d r
\end{aligned}
$$

For any $y^{*} \in[0, Y]$ and any sequence $\left\{y_{i}\right\}_{i=1}^{n}$ with $y_{1}=y *<y_{2}<\ldots<y_{n}=Y$, a repeated application of (A.2) with $y=y_{i}, x=y_{i-1}, i=2, \ldots, n$, yields

$$
p(Y)-p\left(y^{*}\right) \leq-\sum_{i=2}^{n} \int_{r\left(y_{i-1}\right)}^{r\left(y_{i}\right)} u^{\prime}\left(w+L-I+y_{i-1}-r\right) d r
$$

\footnotetext{
${ }^{4}$ If $r\left(x_{2}\right)>w+L-I+x_{1}$, the desired result, namely $r\left(x_{2}\right) \geq r\left(x_{1}\right)$, is trivial
} 
Further, a precisely parallel argument, based on incentive compatibility relative to upward deviations in reports, yields

$$
p(Y)-p\left(y^{*}\right) \geq-\sum_{i=1}^{n-1} \int_{r\left(y_{i-1}\right)}^{r\left(y_{i}\right)} u^{\prime}\left(w+L-I+y_{i}-r\right) d r
$$

Given that the right-hand sides of (A.3) and (A.4) are just the approximating sums for the Stieltjes integral in (4), the validity of (4) follows immediately. This completes the proof of Proposition 1.

Lemma 9 Define $c_{0}($.$) so that for any y \in[0, Y]$,

$$
c_{0}(y)=c_{0}(0)+\max (0, y-\hat{y}),
$$

where $c_{0}(0)=w-I$ and $\hat{y}=0$ if $w \geq I, c_{0}(0)=0$ and $\hat{y}$ is chosen so that (14) holds with equality if $w<I$. Then $c_{0}(Y)>c(Y)$ for any $c(.) \neq c_{0}($.$) that$ satisfies (14)-(16).

Proof. Suppose that there is a consumption pattern $c($.$) that satisfies (14)-$ (16) and $c(Y) \geq c_{0}(Y)$. Since (16) implies $c(y) \geq c(Y)-Y+y$ for all $y \leq Y$, this pattern satisfies $c(y) \geq c_{0}(Y)-Y+y$ for all $y \leq Y$. By the definition of $c_{0}($.$) , this implies c(y) \geq c_{0}(y)$ for all $y \leq Y$, if $w \geq I$, and $c(y) \geq c_{0}(y)$ for all $y \in[\hat{y}, Y]$, if $w<I$. In the latter case, moreover, (15) implies $c(y) \geq c_{0}(y)$ for all $y \leq \hat{y}$, so if $w<I$ one also has $c(y) \geq c_{0}(y)$ for all $y \leq Y$. Given that $c_{0}($. satisfies (14) with equality, it follows that $c($.$) satisfies (14) only if c()=.c_{0}($.$) .$

Proof of Proposition 3. Given (1), the constant function with value

$$
K:=w-I+\int y d G(y)>0
$$

satisfies (14)-(16) so the set of functions $c($.$) satisfying these constraints is$ nonempty. Lemma 9 implies that on this set the objective function (13) is bounded above by $u\left(c_{0}(Y)\right.$ ). Therefore the objective function (13) has a supremum $u^{*}$ on this set. Given that the constant function with value $K$ satisfies (14)-(16), $u^{*} \geq u(K)-u^{\prime}(K) Y$, so $u^{*}$ is finite. Let $\left\{c^{k}().\right\}$ be a sequence of functions satisfying (14)-(16) such that $u\left(c^{k}(Y)\right)-\int u^{\prime}\left(c^{k}(y)\right) G(y) d y$ converges to $u^{*}$ as $k$ goes out of bounds. Using the sequence $\left\{c^{k}().\right\}$, I construct a "limit function" $c($.$) that satisfies (14)-(16) and u(c(Y))-\int u^{\prime}(c(y)) G(y) d y=u^{*}$.

I use a variation of the well known argument that is used for Helly's Selection Theorem (see, e.g., Billingsley (1968). First for any $y>0$, the sequence $\left\{c^{k}(y)\right\}$ is uniformly bounded. To see this, note that for any $y>0$ and any $k,(16)$ and (15) imply $c^{k}(z) \geq c^{k}(y)-y$ for $z \leq y$ and $c^{k}(z) \geq 0$ for $z>y$. By (14) it then follows that

$$
c^{k}(y) G(y)-y G(y) \leq w-I+\int z g(z) d z
$$


and hence that

$$
c^{k}(y) \leq y+\frac{\left(w-I+\int z g(z) d z\right)}{G(y)},
$$

uniformly in $k$.

Next, let $\left\{y_{i}\right\}$ be a countable dense subset of the interval $(0, Y]$. Given that for each $i$ the sequence $\left\{c^{k}\left(y_{i}\right)\right\}$ is uniformly bounded, taking subsequences if necessary, one may suppose that each of the sequences $\left\{c^{k}\left(y_{i}\right)\right\}, i=1,2, \ldots$, converges to a limit $c\left(y_{i}\right)$. The function $c($.$) that is thus defined on the set \left\{y_{i}\right\}$ may be extended to the entire interval $[0, Y]$ by setting

$$
c(y)=\sup \lim _{n} c\left(y_{i_{n}}\right)
$$

where the supremum is taken over all sequences $\left\{y_{i_{n}}\right\}$ in $\left\{y_{i}\right\}$ that converge to $y$. Given that the functions $c^{k}(),. k=1,2, \ldots$, all satisfy (15) and (16), one easily verifies that the function $c($.$) also satisfies (15) and (16), and that$ $c(y)=\lim _{k} c^{k}(y)$ for any continuity point y of $c($.$) . By construction, c($.$) is up-$ per semi-continuous. In view of (16) this implies that $c($.$) is left-continuous$ and that any discontinuity point $y$ of $c($.$) satisfies c(y)>\lim _{y^{\prime} \backslash y} c\left(y^{\prime}\right)$. This in turn implies that $c($.$) has at most countably many discontinuity points, so$ the set of continuity points of $c($.$) and hence the set of points on which \left\{c^{k}(y)\right\}$ converges to $c(y)$ and $\left\{u^{\prime}\left(c^{k}(y)\right)\right\}$ converges to $u^{\prime}(c(y))$ has full measure. By Fatou's Lemma, it follows that the validity of (14) for $c^{k}(),. k=1,2, \ldots$, entails the validity of (14) for $c($.$) and moreover that$

$$
\begin{aligned}
& u(c(Y))-\int u^{\prime}(c(y)) G(y) d y \\
= & \lim _{y_{i} \rightarrow Y} \lim _{k \rightarrow \infty} u\left(c^{k}\left(y_{i}\right)\right)-\int \lim _{k \rightarrow \infty} u^{\prime}\left(c^{k}(y)\right) G(y) d y \\
\geq & \lim _{k \rightarrow \infty}\left[u\left(c^{k}(Y)\right)-\int u^{\prime}\left(c^{k}(y)\right) G(y) d y\right]=u^{*}
\end{aligned}
$$

By the definition of $u^{*}$, it follows that $u(c(Y))-\int u^{\prime}(c(y)) G(y) d y=u^{*}$ so that the supremum of (13) on the set of functions satisfying (14)-(16) is actually attained at $c($.$) , and hence is a maximum.$

Proof of Proposition 4. Suppose first that $c($.$) is not continuous on (0, Y]$. If $c($.$) is not continuous at Y$, then, by (16), one must have $\lim _{y^{\prime}}{ }_{Y} c\left(y^{\prime}\right)>c(Y)$, and an increase in $c(Y)$ will raise the value of (13) without affecting the validity of (14) - (16). If $c($.$) is not continuous on (0, Y)$, then, again by (16), there exists $y \in(0, Y)$ such that $\lim _{y^{\prime}}>y=\left(y^{\prime}\right)>\lim _{y^{\prime} \backslash y} c\left(y^{\prime}\right)$, and, by the strict monotonicity of $u^{\prime}(),. \lim _{y^{\prime} \succ y} u^{\prime}\left(c\left(y^{\prime}\right)\right)<\lim _{y^{\prime} \backslash y} u^{\prime}\left(c\left(y^{\prime}\right)\right)$. But then a small reduction in $c\left(y^{\prime}\right)$ for $y^{\prime}$ belonging to a small interval to the left of $y$, combined with a suitably chosen small increase in $c\left(y^{\prime}\right)$ for $y^{\prime}$ belonging to a small interval to the right of $y$, will raise the value of (13) without affecting the validity of 
(14) - (16). The assumption that $c($.$) is not continuous on (0, Y]$ thus leads to a contradiction and must be false.

To prove that $c(Y)>0$, I note that, by standard arguments, there exists a Lagrange multiplier $\mu$ such that if $c($.$) maximizes (13) under the constraints$ (14)-(16), then $c($.$) also maximizes$

$$
u(c(Y))-\int_{0}^{Y} u^{\prime}(c(y)) G(y) d y+\mu\left(K-\int_{0}^{Y} c(y) g(y) d y\right)
$$

under the constraints (15) and (16); the constant $K$ in (A.7) is again given by (A.6). Since both $u($.$) and -u^{\prime}($.$) are strictly increasing functions, \mu$ must be strictly positive. For any $\varepsilon>0$, consider the consumption pattern $\hat{c}_{\varepsilon}($.$) such$ that $\hat{c}_{\varepsilon}(y)=c(y)$ if $y \leq Y-\varepsilon$ and $\hat{c}_{\varepsilon}(y)=c(Y-\varepsilon)+y-Y+\varepsilon$ if $y \geq Y-\varepsilon$. Clearly, $\hat{c}_{\varepsilon}($.$) satisfies (15)$ and $(16)$, and so does $\hat{c}_{\varepsilon}^{\lambda}()=.(1-\lambda) c()+.\lambda \hat{c}_{\varepsilon}($.$) for$ any $\lambda \in[0,1]$. It follows that for any $\varepsilon>0$ the derivative

$$
\frac{d}{d \lambda}\left[u\left(\hat{c}_{\varepsilon}^{\lambda}(Y)\right)-\int_{0}^{Y}\left(u^{\prime}\left(\hat{c}_{\varepsilon}^{\lambda}(y)\right) G(y)+\mu \hat{c}_{\varepsilon}^{\lambda}(y) g(y)\right) d y\right]
$$

must be nonpositive at $\lambda=0$, and one must have

$$
\begin{aligned}
& u^{\prime}(c(Y))(\varepsilon+c(Y-\varepsilon)-c(Y)) \\
& \quad-\int_{Y-\varepsilon}^{Y}\left(u^{\prime \prime}(c(y)) G(y)+\mu g(y)\right)(y-Y+\varepsilon+c(Y-\varepsilon)-c(y)) d y \leq 0 .
\end{aligned}
$$

Since $u^{\prime \prime}(c(y))<0$ for all $y$ and, by (16), $0 \leq y-Y+\varepsilon+c(Y-\varepsilon)-c(y) \leq$ $\varepsilon+c(Y-\varepsilon)-c(Y)$ for all $y \in[Y-\varepsilon, Y]$, it follows that ,

$$
\left[u^{\prime}(c(Y))-\mu(1-G(Y-\varepsilon))\right](\varepsilon+c(Y-\varepsilon)-c(Y)) \leq 0
$$

for any $\varepsilon>0$. However if $\varepsilon$ is close to zero, $\left[u^{\prime}(c(Y))-\mu(1-G(Y-\varepsilon))\right]>0$, so (A.8) must imply $c(Y)=\varepsilon+c(Y-\varepsilon) \geq \varepsilon>0$. For $y=Y-\varepsilon$ close to $Y$, this in turn yields $c(y)=c(Y)+y-Y$. The second statement of the proposition is thereby proved.

Proof of Proposition 5. To prove Proposition 5, I note that the consumption pattern $c($.$) has a Lebesgue decomposition$

$$
c(.)=c_{A}(.)+c_{S}(.)+c_{D}(.)
$$

into an absolutely continuous function $c_{A}($.$) , a singular, continuous function$ $c_{S}($.$) , and a jump function c_{D}($.$) . This follows from the observation that the$ repayment function $r($.$) that corresponds to c($.$) is nondecreasing and therefore$ has a Lebesgue decomposition

$$
r(.)=r_{A}(.)+r_{S}(.)+r_{D}(.)
$$


into an absolutely continuous function $r_{A}($.$) , a singular, continuous function$ $r_{S}($.$) , and a jump function r_{D}($.$) . These three functions are all nondecreasing;$ moreover there is no loss of generality in assuming that $r_{S}(0)=r_{D}(0)=0$. The corresponding decomposition of $c($.$) is given by setting c_{A}(y) \equiv y-r_{A}(y)$, $c_{S}(y) \equiv-r_{S}(y)$, and $c_{D}(y) \equiv-r_{D}(y)$; the continuity of $c($.$) established in$ Proposition 4 implies $c_{D}(y) \equiv-r_{D}(y) \equiv 0$. As for $c_{S}($.$) and c_{A}($.$) , the mono-$ tonicity of $r_{S}($.$) and r_{A}($.$) implies that c_{S}($.$) is nonincreasing and that the$ (Radon-Nikodym) derivative $v($.$) of c_{A}($.$) satisfies$

$$
v(y) \leq 1 \text { for all } y \in[0, Y] .
$$

Now let $\mu>0$ again be the Lagrange multiplier in (A.7) and consider the control problem

$$
\max _{F(.), f(.)}\left[u(c(Y)+F(Y))-\int_{0}^{Y}\left(u^{\prime}(c(y)+F(y)) G(y)+\mu(c(y)+F(y)) g(y)\right) d y\right]
$$

with the constraints

$$
\begin{gathered}
c(y)+F(y) \geq 0 \text { for all } y \in[0, Y], \\
\frac{d F}{d y}=f(y) \text { for all } y \in[0, Y],
\end{gathered}
$$

and

$$
f(y) \leq 1-v(y) \text { for all } y \in[0, Y] .
$$

I claim that the pair $\left(F_{0}(),. f_{0}().\right)$ with $F_{0}(y) \equiv f_{0}(y) \equiv 0$ solves this control problem. Given (A.9), clearly $\left(F_{0}(),. f_{0}().\right)$ satisfies the constraints (A.11)(A.13). Moreover for any pair $(F(),. f()$.$) that satisfies (A.11)-(A.13) one$ easily finds that the consumption pattern $\hat{c}_{F}():.=c()+.F($.$) satisfies (15), and,$ for any $y, z \in[0, Y]$ such that $y \geq z$,

$$
\begin{aligned}
\hat{c}_{F}(y)-\hat{c}_{F}(z) & =c(y)-c(z)+F(y)-F(z) \\
& =\int_{z}^{y} v(x) d x+c_{S}(y)-c_{S}(z)+\int_{z}^{y} f(x) d x \\
& \leq \int_{z}^{y} v(x) d x+\int_{z}^{y}(1-v(x)) d x \\
& \leq y-z,
\end{aligned}
$$

i.e. $\hat{c}_{F}($.$) also satisfies (16). Given that c($.$) maximizes (A.7) under the con-$ straints (15)-(16), it follows that

$$
\begin{aligned}
& u(c(Y))-\int_{0}^{Y}\left(u^{\prime}(c(y)) G(y)+\mu c(y) g(y)\right) d y \\
\geq & u\left(\hat{c}_{F}(Y)\right)-\int_{0}^{Y}\left(u^{\prime}\left(\hat{c}_{F}(y)\right) G(y)+\mu \hat{c}_{F}(y) g(y)\right) d y \\
= & u(c(Y)+F(Y))-\int_{0}^{Y}\left(u^{\prime}(c(y)+F(y)) G(y)+\mu(c(y)+F(y)) g(y)\right) d y
\end{aligned}
$$


for any pair $(F(),. f()$.$) satisfying (A.11)-(A.13), and hence that \left(F_{0}(),. f_{0}().\right)$ maximizes (A.10) under the constraints (A.11)-(A.13).

Pontryagin's maximum principle now implies the existence of a continuously differentiable real-valued function $\psi($.$) such that for all y \in[0, Y]$,

$$
\begin{gathered}
\frac{d \psi}{d y}(y) \leq u^{\prime \prime}(c(y)) G(y)+\mu g(y), \text { with equality unless } c(y)=0, \\
f_{0}(y)=0 \in \arg \max _{f \leq 1-v(y)} \psi(y) f ;
\end{gathered}
$$

moreover, since $c(Y)>0$, one has the transversality conditions

$$
\psi(Y)=u^{\prime}(c(Y))
$$

and

$$
\psi(0) \leq 0, \text { with equality unless } c(0)=0 .
$$

Note that (A.14) and (A.16) are the same as (17) and (19). Further, (A.15) implies

$$
\psi(y) \geq 0, \text { with equality unless } v(y)=1 .
$$

To establish (18), it is therefore necessary and sufficient to show that if $\psi(y)>0$, then in a neighbourhood of $y$ the singular component $c_{S}($.$) of c($.$) is constant.$ For this purpose, note that $c_{A}($.$) , the absolutely continuous component of c($.$) ,$ satisfies (15) and (16), and so does $\hat{c}_{\lambda}()=.(1-\lambda) c()+.\lambda c_{A}($.$) , for any$ $\lambda \in[0,1]$. Given that $c($.$) maximizes (A.7) subject to (15) and (16), it follows$ that the derivative

$$
\frac{d}{d \lambda}\left[u\left(\hat{c}_{\lambda}(Y)\right)-\int_{0}^{Y}\left(u^{\prime}\left(\hat{c}_{\lambda}(y)\right) G(y)+\mu \hat{c}_{\lambda}(y) g(y)\right) d y\right]
$$

must be nonpositive at $\lambda=0$, or that

$$
u^{\prime}(c(Y))\left(-c_{S}(Y)\right)-\int_{0}^{Y}\left(u^{\prime \prime}(c(y)) G(y)+\mu g(y)\right)\left(-c_{S}(y)\right) d y \leq 0 .
$$

Upon adding $\int_{0}^{Y} \frac{d \psi}{d y}\left(-c_{S}(y)\right) d y-\psi(Y)\left(-c_{S}(Y)\right)+\int_{0}^{Y} \psi(y) d\left(-c_{S}(y)\right)=0$ to the left-hand side and rearranging terms, using (A.16) and the fact that $c_{S}(0)=0$, one can rewrite (A.19) as

$$
\begin{gathered}
\int_{0}^{Y} \psi(y) d\left(-c_{S}(y)\right) \\
-\int_{0}^{Y}\left[u^{\prime \prime}(c(y)) G(y)+\mu g(y)-\frac{d \psi}{d y}(y)\right]\left(-c_{S}(y)\right) d y \leq 0 .
\end{gathered}
$$


Given that $c_{S}($.$) is nonincreasing and c_{S}(0)=0,(\mathrm{~A} .14)$ implies that the last term on the left-hand side is nonnegative, hence

$$
\int_{0}^{Y} \psi(y) d\left(-c_{S}(y)\right) \leq 0
$$

Since $c_{S}($.$) is nonincreasing, (A.21) can only hold if c_{S}($.$) is constant in the$ neighbourhood of any point $y$ satisfying $\psi(y)>0$. (18) is thereby proved. As for (20), this follows trivially from (A.15) and (A.18).

To complete the proof of Proposition 5, assume that $u^{\prime \prime}($.$) is a strictly in-$ creasing function. Then $-u^{\prime}($.$) is a strictly concave function, and (13) defines$ a strictly concave functional on the set of consumption plans $c($.$) . Since the$ set of plans satisfying (14)-(16) is convex, the optimal $c($.$) is unique up to a$ set of measure zero; given the continuity property established in Proposition 4 , the optimal $c($.$) in fact is unique up to the possible discontinuity at y=0$. Sufficiency of Pontryagin's conditions for characterizing this optimal $c($.$) follows$ as in Theorem 1, p. 141, of Mangasarian (1966).

Proof of Proposition 6. (a) Given the consumption pattern specified in $(21)$, set $\mu=\hat{\mu}(\delta)$ and $\psi()=.\hat{\psi}(., \delta)$ where

$$
\hat{\mu}(\delta)=\frac{1+\delta \hat{y}-e^{-\delta(Y-\hat{y}+c(0))}}{Y-\hat{y}}
$$

and for any $y \in[0, Y]$,

$$
\begin{gathered}
\hat{\psi}(y, \delta)=0 \quad \text { if } y<\hat{y}, \\
\hat{\psi}(y, \delta)=\left[(1+\delta y) e^{-\delta(y-\hat{y}+c(0))}-(1+\delta \hat{y}) e^{-\delta c(0)}+\mu(y-\hat{y})\right] / Y \\
\text { if } y \geq \hat{y} .
\end{gathered}
$$

For $y<\hat{y},(\mathrm{~A} .23)$ implies

$$
\begin{aligned}
\frac{d \psi}{d y}(y) & =\frac{\partial \hat{\psi}}{\partial y}(y, \delta)=0 \leq-\delta^{2} \frac{y}{Y}+\hat{\mu}(\delta) \frac{1}{Y} \\
& =u^{\prime \prime}(0) G(y)+\mu g(y)
\end{aligned}
$$

if $\delta$ is sufficiently small so that $\delta^{2} \hat{y} \leq \hat{\mu}(\delta)$. For $y>\hat{y},(A .24)$ implies

$$
\begin{aligned}
\frac{d \psi}{d y}(y) & =\frac{\partial \hat{\psi}}{\partial y}(y, \delta)=-\delta^{2} e^{-d(y-\hat{y}+c(0))} \frac{y}{Y}+\hat{\mu}(\delta) \frac{1}{Y} \\
& =u^{\prime \prime}(c(y)) G(y)+\mu g(y) .
\end{aligned}
$$

In either case, (17) is verified. For $y>\hat{y},(\mathrm{~A} .24)$ and (A.22), also imply $\lim _{\delta \rightarrow 0} \frac{\partial \hat{\psi}}{\partial y}(y, \delta)=\lim _{\delta \rightarrow 0} \hat{\mu}(\delta) / Y=1 /(Y-\hat{y}) Y$, uniformly in $y$. Hence, if $\delta$ 
is sufficiently small, one has $\psi(y)=\hat{\psi}(y, \delta)>0$ for all $y>\hat{y}$. In combination with (A.23), this shows that the given $c(),. \mu$, and $\psi($.$) also satisfy (18) if \delta$ is sufficiently small. As for (19) and (20), these are obviously implied by (A.22) and (A.24). Part (a) of the proposition therefore follows from the last part of Proposition 5 and the observation that $u^{\prime \prime}(c)=-\delta^{2} e^{-\delta c}$ is strictly increasing in $c$.

(b) To prove part (b), I begin by specifying the critical values $y_{1}(\delta), y_{2}(\delta)$ for the kinks in the consumption pattern $c(., \delta)$. By standard calculus arguments, there exists a unique $z_{1}>1$ such that

$$
e^{z_{1}}=1+z_{1}+z_{1}^{2} .
$$

For any $X \geq 3$, there exists $z_{2}(X) \in(1, X)$ such that

$$
z_{2}(X)=\frac{1-e^{-\left(X-z_{2}(X)\right)}}{X-z_{2}(X)-1} .
$$

(The function $z_{3} \rightarrow f\left(z_{3}\right):=z_{3}+\left(1-e^{-z_{3}}\right) /\left(z_{3}-1\right)$ ranges between $+\infty$ and $3-e^{-2}$ as $z_{3}$ ranges from 1 to 2 , so put $z_{2}(X)=\max _{z_{3} \in f^{-1}(X) \cap[1,2]}(1-$ $\left.e^{-z_{3}}\right) /\left(z_{3}-1\right)$.) For $\delta \geq 3 / Y$, put

$$
y_{1}(\delta)=z_{1} / \delta
$$

and

$$
y_{2}(\delta)=z_{2}(\delta Y) / \delta
$$

From (A.27), one has $\lim _{\delta \rightarrow \infty} y_{1}(\delta)=0$. From (A.26) and the fact that $z_{2}(X)>$ 1 for $X \geq 3$, one also has $\lim _{X \rightarrow \infty}\left(X-z_{2}(X)\right)=1$, so (A.28) implies $\lim _{\delta \rightarrow \infty} \delta\left(Y-y_{2}(\delta)\right)=1$, hence $\lim _{\delta \rightarrow \infty} y_{2}(\delta)=Y$. In particular, one has $0<y_{1}(\delta)<y_{2}(\delta)<Y$ if $\delta$ is sufficiently large.

Let $\delta$ be such that $0<y_{1}(\delta)<y_{2}(\delta)<Y$, and, for any parametrically given $\mu>0$, consider the consumption pattern $\hat{c}(., \delta, \mu)$ such that

$$
\begin{aligned}
\hat{c}(y, \delta, \mu) & =\frac{1}{\delta} \ln \left(\delta y_{1}(\delta)\right)+y-y_{1}(\delta)-\frac{1}{\delta} \ln (\mu / \delta) \text { if } y<y_{1}(\delta), \\
\hat{c}(y, \delta, \mu) & =\frac{1}{\delta} \ln (\delta y)-\frac{1}{\delta} \ln (\mu / \delta) \quad \text { if } y \in\left(y_{1}(\delta), y_{2}(\delta)\right), \\
\hat{c}(y, \delta, \mu) & =\frac{1}{\delta} \ln \left(\delta y_{2}(\delta)\right)+y-y_{2}(\delta)-\frac{1}{\delta} \ln (\mu / \delta) \text { if } y>y_{2}(\delta) .
\end{aligned}
$$

Given the monotonicity of $\hat{c}(., \delta, \mu)$ in $\mu$, there exists a unique $\mu(\delta)$ such that

$$
\int \hat{c}(y, \delta, \mu(\delta)) d G(y)=K
$$

where $K>0$ is again given by (A.6). I claim that for any sufficiently large $\delta$ the consumption pattern $c(., \delta):=\hat{c}(., \delta, \mu(\delta))$ corresponds to an optimal incentivecompatible contract. 
To establish this claim, I first show that if $\delta$ is sufficiently large, then $c(., \delta)$ satisfies the constraints (14)-(16). (14) holds trivially, by the definition of $\mu(\delta)$. As for (15), I note that (A.27)-(A.31) imply

$$
-y_{1}(\delta) \leq c(y, \delta)+\frac{1}{\delta} \ln (\mu(\delta) / \delta) \leq \frac{1}{\delta} \ln (\delta Y)+Y-y_{2}(\delta)
$$

for all $y$ and $\delta$, hence

$$
\lim _{\delta \rightarrow \infty}\left[c(y, \delta)+\frac{1}{\delta} \ln (\mu(\delta) / \delta)\right]=0,
$$

uniformly in $y$. From (A.32) this implies $\lim _{\delta \rightarrow \infty} \frac{1}{\delta} \ln (\mu(\delta) / \delta)=K$ and hence $\lim _{\delta \rightarrow \infty} c(y, \delta)=K$, uniformly in $y$. Since $K>0$, it follows that $c(y, \delta)>0$, confirming (15) for all $y$ if $\delta$ is sufficiently large. Finally, (A.27)-(A.31) imply that $\frac{d c}{d y}=1$ if $y<y_{1}(\delta)$ or $y>y_{2}(\delta)$, and $\frac{d c}{d y}=\frac{1}{\delta y} \leq \frac{1}{\delta y_{1}(\delta)}=\frac{1}{z_{1}}<1$ if $y \in\left(y_{1}(\delta), y_{2}(\delta)\right)$, so $(16)$ holds as well.

To show that $c(., \delta):=\hat{c}(., \delta, \mu(\delta))$ is actually optimal, I specify the costate variable $\psi()=.\hat{\psi}(., \delta)$ where

$$
\begin{gathered}
\hat{\psi}(y, \delta)=\left[(1+\delta y) e^{-\delta\left(y-y_{1}(\delta)\right)}-e^{\delta y_{1}(\delta)}+\delta^{2} y_{1}(\delta) y\right] \frac{\mu(\delta)}{\delta^{2} y_{1}(\delta) Y} \\
\text { if } y<y_{1}(\delta), \\
\hat{\psi}(y, \delta)=0 \quad \text { if } y \in\left(y_{1}(\delta), y_{2}(\delta)\right), \\
\hat{\psi}(y, \delta)=\left[(1+\delta y) e^{-\delta\left(y-y_{2}(\delta)\right)}-1-\delta y_{2}(\delta)+\delta^{2} y_{2}(\delta)\left(Y-y_{2}(\delta)\right)\right] \frac{\mu(\delta)}{\delta^{2} y_{2}(\delta) Y} \\
\text { if } y>y_{2}(\delta) .
\end{gathered}
$$

Given the conditions (A.25)-(A.28) for $y_{1}(\delta)$ and $y_{2}(\delta)$, it is straightforward to check that the consumption pattern $c(., \delta)$, the Lagrange multiplier $\mu(\delta)$, and the costate variable $\psi()=.\hat{\psi}(., \delta)$ defined by (A.33)-(A.35) satisfy conditions (17)-(20). As in part (a) of the proposition, the optimality of the consumption pattern $c(., \delta)$ for any sufficiently high $\delta$ now follows from the last part of Proposition 5.

Proof of Proposition 7. Let $\lim _{c \rightarrow 0} u^{\prime}(c)=\infty$. If $c(y)=0$ for a nonnull set of return levels $y \in[0, Y]$, then the integral in (13) is undefined and one cannot be at a maximum of (13) under the constraints (14)-(16). Therefore one must have $c(y)>0$ for all but a null set of return levels $y \in[0, Y]$. Suppose that, contrary to the first statement of the proposition, $c\left(y^{\prime}\right)=0$ for some $y^{\prime}>0$. Then there exists a sequence $\left\{y^{k}\right\}$ converging to $y^{\prime}$ from below such that the associated sequence $\left\{c\left(y^{k}\right)\right\}$ converges to $c\left(y^{\prime}\right)=0$ monotonically from above and moreover $v\left(y^{k}\right)<1$ for all $k$. By (18), it follows that $\psi\left(y^{k}\right)=0$ for all $k$ 
and hence that $\frac{d \psi}{d y}\left(y^{\prime}\right)=0$, which is incompatible with (17). This proves the first statement of the proposition.

Next impose the stronger assumption that $\lim _{c \rightarrow \infty} u^{\prime \prime}(c) c=-\infty$. To prove the second statement of the proposition, I first show that this assumption implies $\psi(y)>0$ and hence, by $(18), \frac{d c}{d y}=1$ for any $y$ that is sufficiently close to zero. For suppose that this claim is false. Then there exists a sequence $\left\{y^{k}\right\}$ converging to zero such that $\psi\left(y^{k}\right)=0$ for all $k$. Then (18) implies that for each $k, \psi($.$) has a local minimum at y^{k}$, i.e., one must have

$$
\frac{d \psi}{d y}\left(y^{k}\right)=0
$$

for all $k$. Since $\lim _{c \rightarrow \infty} u^{\prime \prime}(c) c=-\infty$ implies $\lim _{c \rightarrow 0} u^{\prime}(c)=\infty$, for each $k$ one must also have $c\left(y^{k}\right)>0$. By (17), it follows that (A.36) entails

$$
u^{\prime \prime}\left(c\left(y^{k}\right)\right) G\left(y^{k}\right)+\mu g\left(y^{k}\right)=0
$$

for all $k$. Since $g($.$) is continuous and strictly positive on [0, Y]$, and moreover $\lim _{k \rightarrow \infty} G\left(y^{k}\right)=0$, it follows that $\lim _{k \rightarrow \infty} u^{\prime \prime}\left(c\left(y^{k}\right)\right)=-\infty$ and hence that $c(0)=\lim _{k \rightarrow \infty} c\left(y^{k}\right)=0$. From (16), this implies $c\left(y^{k}\right) \leq y^{k}$ for all $k$. However upon rewriting (A.37) in the form

$$
u^{\prime \prime}\left(c\left(y^{k}\right)\right) c\left(y^{k}\right) \frac{y^{k}}{c\left(y^{k}\right)} \frac{G\left(y^{k}\right)}{y^{k}}+\mu g\left(y^{k}\right)=0
$$

and using l'Hospital's rule, one also finds that $\lim _{k \rightarrow \infty} u^{\prime \prime}\left(c\left(y^{k}\right)\right) c\left(y^{k}\right)=-\infty$ implies $\lim _{k \rightarrow \infty}\left(y^{k} / c\left(y^{k}\right)\right)=0$ and hence that $y^{k}<c\left(y^{k}\right)$ for any sufficiently large $k$. The assumption that there exists a sequence $\left\{y^{k}\right\}$ converging to zero such that $\psi\left(y^{k}\right)=0$ for all $k$ has thus led to a contradiction and must be false. This proves that $\psi(y)>0$ and $\frac{d c}{d y}(y)=1$ for any $y$ that is sufficiently close to zero.

By Proposition 5, this latter conclusion in turn implies that

$$
0<\psi(y)=\psi(y)-\psi(0) \leq \int_{0}^{y}\left[u^{\prime \prime}(c(0)+x) G(x)+\mu g(x)\right] d x
$$

for any $y$ that is sufficiently close to zero. Now (A.39) implies

$$
-\underline{\mathrm{g}} \int_{0}^{y} u^{\prime \prime}(c(0)+x) x d x \leq \mu \bar{g} y
$$

where $\mathrm{g}$ and $\bar{g}$ are the minimum and the maximum, respectively, of the density $g($.$) on [0, Y]$. If (A.40) is to hold for any $y$ that is sufficiently close to zero, it follows that $-u^{\prime \prime}(c(0)+x) x$ must be uniformly bounded even as $x$ is close to zero. Under the assumption that $\lim _{c \rightarrow 0} u^{\prime \prime}(c) c=-\infty$, this is possible only if $c(0)>0$. This completes the proof of Proposition 7 .

Proof of Proposition 8. Since $\lim _{c \rightarrow 0} c^{\delta-1}=\infty$ and $\lim _{c \rightarrow 0} u^{\prime \prime}(c) c=$ $(\delta-1) c^{\delta-1}=-\infty$, Proposition 7 implies $c(0)>0$ and $\frac{d c}{d y}(y)=1$ for $y$ close to 
zero. Since $w<I,(14)$ implies that $c\left(y^{\prime}\right)<y^{\prime}$ for some $y^{\prime} \in(0, Y]$. By (18) it follows that the set $I=\{y \in(0, Y) \mid \psi(y)=0\}$ has positive measure. Define $y_{1}=\inf I$ and $y_{2}=\sup I$. I claim that in fact $I=\left[y_{1}, y_{2}\right]$, i.e., $I$ is a closed interval. For suppose not. Then there exists $y^{\prime} \in\left[y_{1}, y_{2}\right]$ such that $\psi\left(y^{\prime}\right)>0$. By continuity, $\psi(y)$ must actually be positive on some neighbourhood of $y^{\prime}$; this implies $y^{\prime} \neq y_{1}, y^{\prime} \neq y_{2}$ and hence $y^{\prime} \in\left(y_{1}, y_{2}\right)$. One may therefore define $\bar{y}_{1}=\sup I \cap\left[0, y^{\prime}\right]$ and $\bar{y}_{2}=\inf I \cap\left[y^{\prime}, Y\right]$, and one has $\bar{y}_{1}<\bar{y}_{2}$. For $y \in\left(\bar{y}_{1}, \bar{y}_{2}\right)$, one has $\psi(y)>0$, hence, by (18),

$$
c\left(\bar{y}_{2}\right)=c\left(\bar{y}_{1}\right)+\bar{y}_{2}-\bar{y}_{1} .
$$

Next I note that, again by (18), at any $y \in I$, the function $\psi($.$) has a$ minimum and satisfies $\frac{d \psi}{d y}=0$. By (17) in conjunction with the fact that $c(y)>0$ for all $y$, this implies that

$$
u^{\prime \prime}(c(y)) G(y)+\mu g(y)=0
$$

for all $y \in I$. For the given utility function and distribution function, it follows that

$$
\begin{aligned}
c(y) & =\hat{c}(y, \mu) \text { where } \\
\hat{c}(y, \mu) & =(1-\delta)\left(\frac{y}{\mu}\right)^{\frac{1}{2-\delta}}
\end{aligned}
$$

for all $y \in I$. By continuity, one has $\psi\left(\bar{y}_{i}\right)=0$, hence $\bar{y}_{i} \in I$ for $i=1,2$. From (A.43), one then has

$$
c\left(\bar{y}_{i}\right)=\hat{c}\left(\bar{y}_{i}, \mu\right)
$$

for $i=1,2$. From (A.41) and (A.45), it follows that there is some $y^{\prime \prime} \in\left(\bar{y}_{1}, \bar{y}_{2}\right)$ such that

$$
\frac{\partial \hat{c}}{\partial y}\left(y^{\prime \prime}, \mu\right)=1 .
$$

Given that the function $\hat{c}(., \mu)$ is strictly concave, (A.46) implies

$$
\frac{\partial \hat{c}}{\partial y}(y, \mu)>1
$$

for all $y \leq \bar{y}_{1}$. From (16), (A.45), and (A.46) one then obtains

$$
\begin{aligned}
c(y) & \geq c\left(\bar{y}_{1}\right)-\bar{y}_{1}+y \\
& >\hat{c}\left(\bar{y}_{1}, \mu\right)-\int_{y}^{\bar{y}_{1}} \frac{\partial \hat{c}}{\partial y}(y, \mu) d y \\
& =\hat{c}(y, \mu)
\end{aligned}
$$

for all $y<\bar{y}_{1}$. In view of (A.44), (A.48) implies $u^{\prime \prime}(c(y)) G(y)+\mu g(y) \neq 0$ and hence, by $(17)$, that $\frac{d \psi}{d y} \neq 0$ for all $y<\bar{y}_{1}$. Given that $\psi\left(\bar{y}_{1}\right)=0$, this 
is incompatible with the transversality condition (20) requiring that $\psi(0)=0$. The assumption that the set $I=\{y \in(0, Y) \mid \psi(y)=0\}$ is not a closed interval $\left[y_{1}, y_{2}\right]$ has thus led to a contradiction and must be false.

Given that $I=\left[y_{1}, y_{2}\right]$, the argument just given shows that $c($.$) satisfies$ (A.43) and (A.44) and hence (27) on $\left[y_{1}, y_{2}\right]$. Since $\lim _{y \rightarrow 0} \frac{\partial \hat{c}}{\partial y}(y, \mu)=\infty$, it follows that $y_{1}>0$. For $y \in\left(0, y_{1}\right), \psi(y)>0$ and hence by (18), $\frac{d c}{d y}=1$. Finally, Proposition 4 implies that $y_{2}<Y$. For $y \in\left(y_{2}, Y\right], \psi(y)>0$ and hence, again by (18), $\frac{d c}{d y}=1$. This shows that $c($.$) also satisfies (26) and completes the$ proof of Proposition 8.

\section{References}

[1] Billingsley, P. (1968), Convergence of Probability Measures, Wiley, New York.

[2] Diamond, D. (1984), Financial Intermediation as Delegated Monitoring, Review of Economic Studies 51, 393-414.

[3] Gale, D., and M. Hellwig, Incentive-Compatible Debt Contracts: The OnePeriod Problem, Review of Economic Studies 52, 647-663.

[4] Garino, G., and P.J.Simmons (1998), A Note on Costly State Verification Contracts with Risk Aversion, mimeo, University of York, UK

[5] Hellwig, M. (1998), Financial Intermediation with Risk Aversion, Discussion Paper No. 98-39, SFB 504, University of Mannheim.

[6] Innes, R.D. (1990), Limited Liability and Incentive Contracting with ExAnte Action Choices, Journal of Economic Theory 52, 45-67

[7] Mangasarian, O.L. (1966), Sufficient Conditions for the Optimal Control of Nonlinear Systems, SIAM Journal of Control 4, 139-152.

[8] Townsend, R.M (1979), Optimal Contracts and Competitive Markets with Costly State Verification, Journal of Economic Theory 21, 1-29. 